\title{
DESENVOLVIMENTO DE EQUIPAMENTO DE LABORATÓRIO PARA SIMULAR PCI EM ALTOS-FORNOS*
}

René Lucio Rech ${ }^{1}$

André da S. Machado

Claudia C. T. Barbieri3

Janaina G. M. da S. Machado ${ }^{4}$

Antonio Cezar Faria Vilela ${ }^{5}$

Eduardo Osório 6

\section{Resumo}

A injeção de carvão pulverizado ( $\mathrm{PCl}$ ) é uma técnica altamente utilizada nos altosfornos pelas siderúrgicas brasileiras, buscando a redução do consumo específico de coque por tonelada de gusa. A combustão do carvão pulverizado ocorre em temperaturas elevadas (em torno de $\left.2.000^{\circ} \mathrm{C}\right)$, altas taxas de aquecimento $\left(10^{4} \mathrm{a} 10^{5}\right.$ ${ }^{\circ} \mathrm{C} / \mathrm{s}$ ), tempo de reação de poucos centésimos de segundos, seguida pela gaseificação na presença de $\mathrm{CO}_{2}$. Este trabalho visa apresentar o andamento da construção do simulador da zona de combustão do alto-forno desenvolvido pelo LASID-UFRGS e os estudos previstos nos projetos em andamento, que contemplam também a avaliação de carvões brasileiros que apresentem potencial de substituição de carvões atualmente importados para injeção em altos fornos brasileiros. A partir do levantamento do estado da arte e visitas técnicas a simuladores em operação, foi discutido um projeto moderno, automatizado, de configuração vertical e que possibilitasse a operação tanto em modo one shot como contínuo. Algumas inovações podem ser destacadas, como a possibilidade de alterar o tempo de residência da amostra carbonosa na zona de combustão do simulador, a medição da temperatura com termopares ultra-rápidos (ms), o controle e aquisição de diversos parâmetros termodinâmicos $(P, T, t)$ e avaliação dos produtos sólidos e gasosos resultantes.

Palavras-chave: Carvão pulverizado; Combustíveis; Alto-forno; Zona de combustão.

\section{DEVELOPMENT OF A LAB-SCALE TEST RACK TO EVALUATE FUELS FOR PCI IN BLAST FURNACES}

\section{Abstract}

Pulverized coal injection ( $\mathrm{PCl}$ ) is widely utilized by Brazilian steel makers, aiming a reduction of coke amount needed in the pig iron production. Coal combustion happens at high temperatures of about $2,000^{\circ} \mathrm{C}$, very high heating rates from $10^{4}$ to $10^{5}{ }^{\circ} \mathrm{C} / \mathrm{s}$ and combustion times of 0,02 to $0,05 \mathrm{~s}$, followed by char gasification with $\mathrm{CO}_{2}$. This is a short status report of the design and construction work of a lab-scale rack to evaluate coals for $\mathrm{PCl}$ being built by LASID-UFRGS at Porto Alegre-RS, Brazil. Remarking features of the design are the possibility of changing residence time of the particles at the combustion zone, use of fast-response thermocouples, control and acquisition of thermodynamic parameters $(\mathrm{P}, \mathrm{T}, \mathrm{t})$ and the collection and evaluation of the resulting solid and gas products.

Keywords: Pulverized coal; Fuels, Blast furnace, Combustion zone.

Eng. Químico, MSc, doutorando, PPGEQ, UFRGS, Porto Alegre, RS, Brasil.

Físico, MS, doutorando, PPGEM, UFMG, Belo Horizonte, MG, Brasil.

Química Industrial, MSc, doutoranda, PPGEM, UFRGS, Porto Alegre, RS, Brasil.

Eng. Metalúrgica, PhD, engenheiro sênior, Centro de Tecnologia de Ferrosos, Vale S.A., Belo Horizonte, MG, Brasil.

5 Eng. Metalúrgico, Dr.Ing., professor, PPGEM, UFRGS, Porto Alegre, RS, Brasil.

6 Eng. Metalúrgico, PhD, professor, PPGEM, UFRGS, Porto Alegre, RS, Brasil.

* Contribuição técnica ao 44 Seminário de Redução de Minério de Ferro e Matérias-primas, 15ํ Simpósio Brasileiro de Minério de Ferro e $2^{\circ}$ Simpósio Brasileiro de Aglomeração de Minério de Ferro, 15 a 18 de setembro de 2014, Belo Horizonte, MG, Brasil. 


\section{INTRODUÇÃO}

Nas últimas décadas, a injeção de carvão pulverizado ( $\mathrm{PCl}$ - Pulverized Coal Injection) nas ventaneiras dos altos-fornos vem sendo adotada em níveis crescentes pela indústria siderúrgica mundial com o objetivo de diminuir o consumo específico de coque por tonelada de gusa e aumentar a produtividade dos altos-fornos, com conseqüente redução do custo de produção [6,7]. Atualmente, os níveis de injeção na maioria dos altos-fornos já alcançam valores de 150 a 200 kg/t gusa [3].

A grande dependência da indústria siderúrgica brasileira da importação de carvões coqueificáveis tem levado à adoção desta prática nos altos-fornos brasileiros, utilizando carvões não-coqueificáveis importados, de custo menor do que o dos carvões coqueificáveis.

A disponibilidade de carvões brasileiros não-coqueificáveis com características para uso potencial em $\mathrm{PCl}$ cria oportunidades de estudos para seu uso efetivo, que passam por uma ampla avaliação tecnológica de suas propriedades relacionadas com este uso. A determinação da combustibilidade dos carvões em condições aproximadas das que ocorrem nas ventaneiras dos altos-fornos é, sem dúvida, uma ferramenta importante para determinar o desempenho no processo real de PCI.

Não existem testes padronizados para a determinação do desempenho dos carvões no processo $\mathrm{PCl}$. Por isso, diversas técnicas em escala laboratorial têm sido utilizadas em condições que nem sempre se aproximam das condições reais. Testes de reatividade em balanças termogravimétricas (TGA) e em fornos de queda livre (DTF) têm sido publicados, inclusive com avaliação de carvões brasileiros e biomassas em misturas $[1,5,14,16]$. Mesmo que a combustão não ocorra em condições similares às do $\mathrm{PCl}$, informações relevantes são obtidas referentes aos mecanismos de desvolatilização por pirólise e de combustão de diferentes carvões, de forma comparativa, como taxas de reação e eficiências de combustão [8].

Uma grande variedade de equipamentos de laboratório e de plantas-piloto tem sido construída em diversos países para avaliar o processo $\mathrm{PCl}$ em condições próximas às reais [2,6,9-13,20-22]. Estes simuladores vêm gerando informações para 0 melhor entendimento dos fenômenos, que são utilizadas nos estudos de modelagem dos processos $\mathrm{PCl}$.

Já há anos, o Laboratório de Siderurgia da Universidade Federal do Rio Grande do Sul - LASID/UFRGS vem realizando estudos na busca de viabilizar a inclusão de matérias-primas nacionais nas misturas para $\mathrm{PCI}[4,14,15,17-19]$. Em 2011, iniciouse um projeto de desenvolvimento de um simulador de $\mathrm{PCl}$ em escala de laboratório para possibilitar estudos de combustibilidade de matérias-primas nacionais e importadas, isoladas ou como misturas, com características modernas, aquisição instantânea de dados, flexibilidade quanto a matérias-primas e variação de parâmetros operacionais e obtenção de amostras de produtos sólidos e gasosos da combustão para análise posterior [15]. Este trabalho tem por objetivo mostrar o andamento da construção do simulador da zona de combustão do alto-forno desenvolvido pelo LASID-UFRGS. Apresenta, além do projeto do simulador, características importantes que o diferenciam dos demais.

A partir do final de 2014, com a conclusão da montagem e dos testes préoperacionais, o novo equipamento estará disponível para projetos acadêmicos e estudos específicos para as indústrias siderúrgicas e carboníferas interessadas.

\footnotetext{
* Contribuição técnica ao 44 Seminário de Redução de Minério de Ferro e Matérias-primas, 15ㅇ Simpósio Brasileiro de Minério de Ferro e 2o Simpósio Brasileiro de Aglomeração de Minério de Ferro, 15 a 18 de setembro de 2014, Belo Horizonte, MG, Brasil.
} 


\section{O SIMULADOR DA ZONA DE COMBUSTÃO DO ALTO-FORNO DO LASID- UFRGS}

\subsection{Projeto do Simulador}

No projeto do simulador do LASID-UFRGS, considerou-se como premissa a sua adequação para pesquisas em laboratório das condições de combustão que se aproximem das que ocorrem na injeção nas ventaneiras dos altos-fornos. Para tanto, deveria ter operação fácil, de baixo custo, com grau elevado de automação, possibilidade de variação e controle de parâmeros operacionais, eficiente aquisição de dados, coleta dos produtos sólidos e gasosos da combustão, configuração vertical, injeção one shot do combustível sólido, porém com potencial de conversão futura para injeção contínua dos combustíveis sólidos. Tomou-se por base conceitual do projeto o simulador existente no IEHK/RWTH, Alemanha.

A Figura 1 apresenta o esquema do simulador do LASID-UFRGS. Como os simuladores usados como referência, o equipamento simula a injeção dos combustíveis sólidos desde a ventaneira até a zona de combustão do alto-forno e apresenta as mesmas áreas representativas destas regiões do alto-forno.

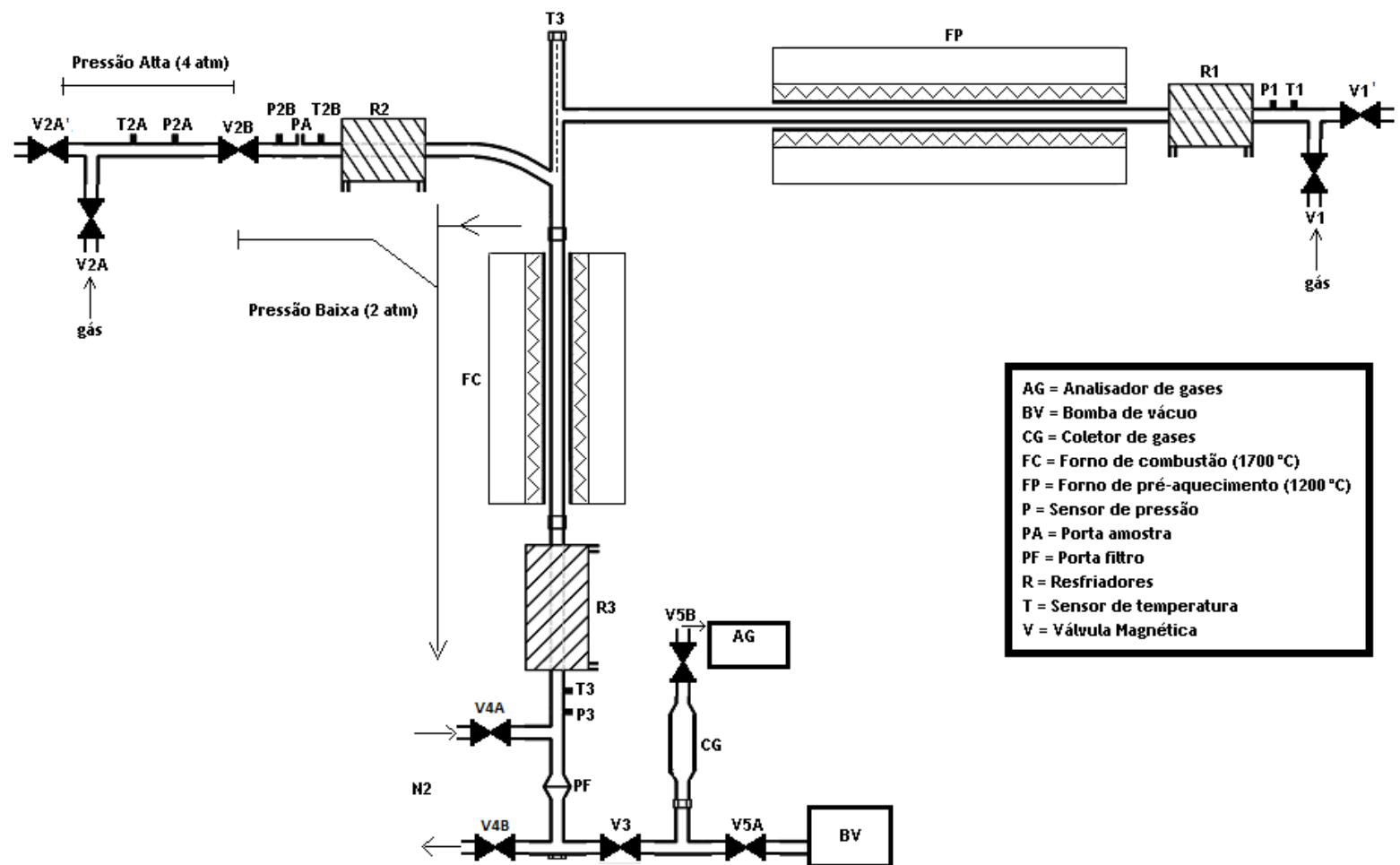

Figura 1 -Esquema do simulador da zona de combustão do LASID-UFRGS

Na versão atual, para injeção one shot do combustível sólido, o simulador apresenta duas regiões com pressões distintas. Na região de pressão mais baixa, onde o combustível sólido a ser testado está previamente depositado na zona fria, ocorre o processo de combustão propriamente dito, desde o aquecimento da amostra, pirólise e combustão do char bem como a coleta dos sólidos. A região de pressão mais alta é apenas um reservatório de gás frio que impulsiona a amostra de combustível sólido depositado na zona fria até a zona de combustão, arrastado pelo fluxo gasoso que ocorre ao se abrir a válvula de separação entre as zonas. Os

\footnotetext{
* Contribuição técnica ao $44^{\circ}$ Seminário de Redução de Minério de Ferro e Matérias-primas, 15은 Simpósio Brasileiro de Minério de Ferro e $2^{\circ}$ Simpósio Brasileiro de Aglomeração de Minério de Ferro, 15 a 18 de setembro de 2014, Belo Horizonte, MG, Brasil.
} 
gases produzidos na combustão em frações de segundo são coletados num reservatório sob vácuo para análise posterior. Futuramente, para conversão do equipamento para operação contínua, serão desenvolvidos sistemas de injeção contínua de sólidos pulverizados em sistema de contra-pressão, amostradores de gases em linha, sistema de amostragem de sólidos (char) com refrigeração e inertização instantânea, bem como a alimentação das misturas gasosas préaquecidas para combustão. O equipamento foi desenhado com o forno de préaquecimento dos gases posicionado horizontalmente (FP) e do forno de combustão posicionado verticalmente (FC). Este arranjo permite medir tanto os perfis térmicos da região de pirólise e pré-aquecimento dos combustíveis, obtendo-se assim dados mais precisos das temperaturas par subsidiar estudos de modelagem do processo. O equipamento foi construído em tubulação de aço inox 310, sch 40, para resistência a pressões de até $10 \mathrm{~atm}(1,0 \mathrm{Mpa})$ e a temperaturas de até $1200{ }^{\circ} \mathrm{C}$ na região de pré-aquecimento. A zona de combustão ocorre em tubo de alumina, com temperaturas previstas de até $1700^{\circ} \mathrm{C}$. O reator vertical de alumina conecta-se aos tubos de aço em encaixes cônicos, sob pressão, pressionado por um sistema de tensionadores e molas helicoidais para evitar vazamentos e poder compensar dilatações ocorridas no processo de aquecimento. O equipamento é dotado de sensores de pressão e temperatura $(P, T)$, que controlam as variáveis de entrada, a eficiência dos resfriadores e fornecem dados a serem coletados durante os ensaios. Os sensores de pressão $(P)$ possuem tempo de resposta inferior a $1 \mathrm{~ms}$; espera-se com isso medir os tempos de residência da amostra de combustível no interior do forno de combustão (zona de combustão). Com a mesma finalidade, serão utilizados termopares ultra-rápidos, com constante de tempo $(63,2 \%$ da T) inferior a $1 \mathrm{~ms}$.

A partir da alteração dos parâmetros de pressão nas regiões de alta e de baixa pressão e do diâmetro do reator de alumina, pode-se variar o tempo de residência da amostra na zona de reação. Uma série de válvulas (V) configuradas sob rotinas automatizadas permite um controle preciso dos intervalos de tempos constituintes das simulações. Foram utilizadas válvulas de alto desempenho, com tempo de resposta inferior a $5 \mathrm{~ms}$. Os gases e particulados resultantes da combustão são filtrados num filtro sinterizado de bronze. Uma bomba de vácuo é responsável pela evacuação do simulador, durante o ciclo de purga, e do coletor de gases. Os gases sãocoletados num reservatório para posterior análise. Quando houver interesse na análise do char recolhido no filtro, as válvulas V4A e V4B são acionadas, permitindo a passagem de $\mathrm{N}_{2}$, que irá resfriar a amostra e protegê-la de reações secundárias de oxidação.

O procedimento do teste de simulação one shotocorre com as seguintes etapas:

-pré-aquecimento dos fornos;

- pressurização e ajuste do sistema de compensação de vazamentos;

- purga com o gás do ensaio;

- evacuação do equipamento e do sistema de coleta de gases;

- colocação do combustível no porta amostra;

-pressurização parcial do equipamento;

-abertura e fechamento da válvula do amostrador;

-pressurização completa do simulador;

-realização do ensaio;

-coleta dos gases e resfriamento e remoção do char;

-análise dos gases;

- análise do char.

* Contribuição técnica ao $44^{\circ}$ Seminário de Redução de Minério de Ferro e Matérias-primas, 15은 Simpósio Brasileiro de Minério de Ferro e 2o Simpósio Brasileiro de Aglomeração de Minério de Ferro, 15 a 18 de setembro de 2014, Belo Horizonte, MG, Brasil. 


\subsection{Características do Simulador}

O simulador de $\mathrm{PCI}$ do LASID-UFRGS apresenta algumas características importantes para possibilitar ampla variedade de testes experimentais com obtenção de dados de boa qualidade. Dentre elas, pode-se destacar:

- A possibilidade de variar o tempo de residência da amostra carbonosa na zona de combustão do simulador e seu rápido resfriamento e inertização no filtro de coleta, permitindo assim estudar-se a evolução da combustão com o tempo, além de poder-se ajustar este tempo ao valor estimado para AFs em estudo;

- A aquisição rápida, em tempo real, dos dados termodinâmicos de temperatura e pressão $(\mathrm{P}, \mathrm{T}, \mathrm{t})$, o que permitirá conduzir estudos, com maior precisão, sobre as reações de gaseificação e combustão ocorridas no interior do simulador;

- A possibilidade de avaliar diferentes combustíveis sólidos isoladamente ou em misturas, como carvões de diferentes ranks, desde alto voláteis até antracíticos, combustíveis renováveis sólidos como madeira, resíduos vegetais, carvões vegetais e mesmo resíduos industriais combustíveis, como plásticos reciclados.

- A possibilidade de variação dos parâmetros operacionais como natureza, granulometria e massa de sólidos alimentados, composição da mistura gasosa, temperaturas e pressões das zonas de pré-aquecimento e de combustão, tempos de residência na zona de combustão traz grande flexibilidade aos testes e estudos que podem ser realizados com o auxílio do simulador.

\section{ETAPAS DO DESENVOLVIMENTO DO SIMULADOR.}

O projeto do simulador de PCI do LASID-UFRGS foi iniciado em 2012 e vem ocorrendo de acordo com as etapas a seguir descritas.

Etapas já executadas:

-Revisão bibliográfica sobre os parâmetros relevantes e fenômenos envolvidos na combustão de finos de carvão sob as condições prevalentes na zona de combustão do AF.

- Estudo sobre o estado da arte da injeção de combustíveis sólidos através de simuladores da zona de combustão do AF.

- Realização de visitas técnicas a simuladores em operação.

- Definição do projeto conceitual de um simulador mais adequado às pesquisas de combustão em escala de laboratório acadêmico, que melhor represente as condições de injeção em AFs.

- Projeto executivo e construção do simulador, definição e aquisição dos equipamentos auxiliares e instrumentação: fornos, termopares, medidores de pressão, controladores, analisador de gases, sistema de mistura e controle de gases e estruturas de suporte.

Etapas em execução:

- Montagem mecânica e testes pré-operacionais : março a setembro de 2014

- Realização dos testes iniciais de validação do simulador com carvões pulverizados e o estabelecimento dos protocolos de operação: agosto a dezembro de 2014.

Para 2015, estão programados estudos de combustilidade para uso em $\mathrm{PCl}$ com carvões nacionais beneficiados, com carvões importados, com misturas de carvões e com misturas de carvões com outros combustíveis e biocombustíveis. Em paralelo, será iniciado o projeto de conversão para o modo de operação contínua do simulador e da adequação para injeção de outros gases reativos e para coleta online de amostras dos produtos sólidos e gasosos da combustão.

* Contribuição técnica ao $44^{\circ}$ Seminário de Redução de Minério de Ferro e Matérias-primas, 15ํ Simpósio Brasileiro de Minério de Ferro e $2^{\circ}$ Simpósio Brasileiro de Aglomeração de Minério de Ferro, 15 a 18 de setembro de 2014, Belo Horizonte, MG, Brasil. 


\section{CONCLUSÃO}

A partir de 2015, o LASID-UFRGS disporá de um equipamento moderno e versátil em escala de bancada capaz de avaliar as características de combustão de carvões minerais e de outros combustíveis sólidos quanto à sua adequação para uso na injeção pulverizada em altos-fornos siderúrgicos.

\section{Agradecimentos}

Os autores agradecem ao $\mathrm{CNPq} /$ Rede Carvão pelo financiamento do projeto e à VALE SA pelo suporte financeiro à infraestrutura para a instalação do equipamento.

\section{REFERÊNCIAS}

1 Al-Omari Y. Influence of coal ash and process conditions on coal/char reactivity for PCl into BF. PhD thesis, The University of New South Wales, Sydney, Australia, 2005.

2 Assis PS. Einblasen von Holzkohle in den Holzkohlehochofen. Dr. Ing. Diss., lehk RA, 1991.

3 Babich A, Senk D, Gudenau HW, Mavrommatis K, Mainz GA. Ironmaking, 2008.

4 Barbieri CCT. Estudo de misturas de carvões e biomassa visando a combustão em alto-forno. Dissertação de mestrado. PPGE3M-UFRGS, Porto Alegre, 2013.

5 Borrego, A.G. \& Alvarez, D. Comparison of chars obtained under oxy-fuel and conventional pulverized coal combustion atmospheres. Energy \& Fuels, 2007 ; $21: 3171-9$.

6 Carneiro RTS, Giudice FDP, Castro JB. Combustibilidade de Carvões para Injeção em Altos-fornos. Relatório Final de Estudo de P\&D, Centro de Pesquisa e Desenvolvimento da Usiminas, Ipatinga-MG, maio, 1997.

7 Carpenter AM. Use of pci in blast furnaces. CCC/116, London, UK, IEA Clean Coal Centre, 66 p., set. 2006a.

8 Carpenter AM. Coal quality assessment -the validity of empirical tests. IEA Clean Coal Center, London, 2006b.

9 Gudenau HW. Coal in Metallurgy. III Congresso Brasileiro de Carvão Mineral, Gramado, Brasil, 21-24 Aug. 2011.

10 Gudenau HW. et alii - Investigation of Pyrolysis and Combusion of Coal Powder by Simulation of the Blast Furnace Injection. RWTH Aachen, 1987.

11 Haywood, R.J., McCarthy, M.J., Truelove, J.S., Mason, M.B. \& Thomson, A.D. An experimental and theoretical investigation of pulverised coal combustion in blast furnaces. Fourth Australian Flame Days, Adelaide, 1995.

12 Khairil D, Katsuya N, Naruse I. Fundamental reaction characteristics of pulverized coal at high temperature. ISIJ International, 2001 ; 41(2) : 136-41.

13 Lu L, Sahajwalla V, Harris D. Coal char reactivity and structural evolution during combustion - factors influencing blast furnace pulverized coal injection operation. Metallurgical and Materials Transactions, 2001; 32b : 811-20.

14 Machado AS, Pohlmann JG, Vilela ACF, Osório E. Comparação da estrutura e reatividade de chars obtidos em forno DTF e simulador da zona de combustão do alto-forno. Artigo Completo: In: Anais do III Congresso Brasileiro de Carvão Mineral, Gramado, 2011.

15 Machado AS, Rech RL, Machado JGMS, Vilela ACF, Osório E. Construção de um simulador da zona de combustão do alto-forno para a avaliação de

* Contribuição técnica ao $44^{\circ}$ Seminário de Redução de Minério de Ferro e Matérias-primas, 15오 Simpósio Brasileiro de Minério de Ferro e 2ํ Simpósio Brasileiro de Aglomeração de Minério de Ferro, 15 a 18 de setembro de 2014, Belo Horizonte, MG, Brasil. 
combustíveis sólidos e líquidos. In : Anais do IV Congresso Brasileiro de Carvão Mineral, p. 304-18, Gramado, 2013.

16 Machado JGMS. Estudo de Reatividade e Combustão de Carvões Minerais, Carvão Vegetal e Misturas. Porto Alegre, 2009. Tese (Doutorado em Engenharia) - Escola de Engenharia, Universidade Federal do Rio Grande do Sul, 2009.

17 Osório E, Gomes MLI, Vilela ACF, Kalkreuth W. Estudo da Utilização de Carvões Gaúchos no processo de injeção de carvão pulverizado em altosfornos. 4th IAS Ironmaking Conference, San Nicolas, 2003.

18 Osório E, Gomes MLI, Vilela ACF, Kalkreuth W, Almeida Borrego AG, Alvarez D. Evaluation of Petrology and Reactivity of Coal Blends for Use in Pulverized Coal Injection (PCI). International Journal of Coal Geology, 2006 ; 68 : 14-29.

19 Pohlmann JG, Borrego AG, Vilela ACF, Osório E. Avaliação da combustibilidade de carvões e biomassas pré-tratadas. In : Anais do IV Congresso Brasileiro de Carvão Mineral, p. 319-27, Gramado, 2013.

20 Reis HMB, Seshadri V, Castro JB, Ulhôa MB. Influência do rank e da composição maceral de carvão pulverizado na eficiência de combustão no raceway do alto-forno. In: XXXIII Seminário de Redução de Minério de Ferro e Matérias-Primas e IV Simpósio Brasileiro de Minério de Ferro da ABM, Ouro Preto, MG, p.467-76, 2003.

21 Ueno H, Yamaguchi K, Tamura K.Coal combustion in the raceway and tuyere of a blast furnace, ISIJ International, 1993 ; 33(6) : 640-5.

22 Wu L, Paterson N, Dugwell DR, Kandiyoti R. Simulation of blastfurnace tuyere and raceway conditions in a wire mesh reactor: extents of combustion and gasification. Energy \& Fuels, 2007 ; 21 : 2325-34.

* Contribuição técnica ao $44^{\circ}$ Seminário de Redução de Minério de Ferro e Matérias-primas, 15o Simpósio Brasileiro de Minério de Ferro e 2o Simpósio Brasileiro de Aglomeração de Minério de Ferro, 15 a 18 de setembro de 2014, Belo Horizonte, MG, Brasil. 